Open Access

\title{
Exploring regulations and scope of the Internet of Things in contemporary companies: a first literature analysis
}

\author{
Giuseppe Russo ${ }^{1}$, Bruno Marsigalia ${ }^{1}$, Federica Evangelista ${ }^{1 *}$, Matteo Palmaccio ${ }^{1}$ and Marina Maggioni ${ }^{2}$
}

\author{
* Correspondence: \\ f.evangelista@unicas.it \\ 'Department of Business and Law, \\ University of Cassino and Southern \\ Lazio, Cassino, Italy \\ Full list of author information is \\ available at the end of the article
}

\begin{abstract}
Purpose: The Internet of Things is the networked connection of people, processes, data and things, which together are able to achieve more relevant and valuable connections than ever before. In this framework, every aspect of economic and social life will be related via sensors and software to an Internet of Things platform, and the resulting data continuously flow into big data at every node. In order to provide a better understanding of this smart infrastructure, the aim of this paper is to investigate the Internet of Things through a revised theory of the topic, examining the period from 1985 to 2015, with the purpose of identifying the new opportunities offered by the Internet of Things to the economic-system and learn how to manage them.

Design/methodology/approach: The Internet of Things was analysed by applying a qualitative method with a single method approach to the existing literature. We developed the research by studying national and international literature and, by running four key words (Internet of Things, Energy Internet, Communications Internet and Logistics Internet) through two databases (Google Scholar and EBSCO), we obtained a range of scientific contributions that could complement the literature review. The research sources are of a secondary nature.
\end{abstract}

Originality/value: The Internet of Things can connect everything to everyone in an integrated global network. This network promises efficiency and social and individual benefits by quantifying and monitoring previously measureless qualities. The analysis of the literature review on the Internet of Things is, therefore, a way to fully appreciate the characteristics, opportunities, possible applications, risks and potential issues linked to the topic.

Practical implications: Through our theory, our aim is to contribute towards identifying the main features, fields of application and corresponding potential applications for the Internet of Things, offering new ideas for further analysis.

Keywords: Big data, Internet of Everything, Internet of Things, Logistics Internet, Communication Internet, Energy Internet, Companies

\section{包 Springer}

C 2015 Russo et al. Open Access This article is distributed under the terms of the Creative Commons Attribution 4.0 International License (http://creativecommons.org/licenses/by/4.0/), which permits unrestricted use, distribution, and reproduction in any medium provided you give appropriate credit to the original author(s) and the source, provide a link to the Creative Commons license, and indicate if changes were made. 


\section{Background}

Introduction

Advances in the areas of smart device-embedded systems, computing, and networking (Trequattrini et al. 2012a) are leading to the creation of an infrastructure composed of millions of heterogeneous devices.

These devices, or "smart things", will not simply convey but process information and form advanced collaborations, in order to define the infrastructure of the "Internet of Things". IoT, together with the other emerging internet developments, such as Energy Internet, Communications Internet and Logistics Internet, are the mainstay of digital economy and digital society and the foundation for the future knowledge basedeconomy (Foray 2004) and innovation society.

This infrastructure will be integrated with the environment and, in particular, with enterprise systems. IoT (Atzori et al. 2010) developments show that we will have over 200 billion connected sensor devices by the year 2020, and these will be the main drivers for further IoT advancements.

Electronic sensors are now ubiquitous, creating new types and greater amounts of high-quality information, which, in turn, open new opportunities for creating services that will bring tangible benefits to society, the environment, the economy and to individual human beings.

Sensors are used in many different ways. These range from the real-time monitoring of water quality in oceans through sensors connected to buoys that send information over general packet radio services (GPRS) networks, to monitoring goods being shipped around the world, and smart power grids that create conditions for more rational planning and consumption in production processes, and can all be achieved by means of microchips implanted in objects that communicate with each other. Retailers can tag individual objects using radio frequency identifiers (RFID) to solve a number of problems all at once. Examples of the areas of application include accurate inventories, control of losses and unattended walk-through point-of-sale terminals. Warehouses can become fully automatic, with items being checked in and out and orders automatically passed to suppliers. Items in transit make intelligent decisions about their routing based on information received from readers and positioning systems. Weather forecasting infrastructure can work with in-ground sensors and irrigation-control software. Irrigation systems can be activated on the basis of intelligent decisions involving the level of moisture in the soil and the likelihood of precipitations. Roadside sensors can detect the flow of cars with RFID-based automatic motorway toll payment tags and provide traffic reports. All sorts of things can give their location to their owners, including keys, wallets, glasses, jewellery and tools. Some IoT-type applications are not new. Among these are toll payment tags, security access key cards, devices to track stolen cars and various types of identity tags for retail goods and livestock.

The line between real and virtual world is blurring and will change the way we design, deploy and use services.

In order to gain a greater understanding of the phenomenon of the Internet of Things, its characteristics and the associated opportunities, possible applications, risks and issues, in our paper, we have started by carrying out an accurate review of the scientific contributions produced over the period between 1985 and 2015 for economic and corporate purposes. 
The research approach adopted is based on qualitative methodology, using a singlemethod approach. The study was conducted by analysing the scientific contribution produced for economic and corporate purposes over the period from 1985 to 2015, using two database and four key words. All the scientific contributions on the theme of Internet of Things were collected, searched and analysed, in order to prepare an updated theory of the topic.

The structure of the paper is the following. After the "Background" section, the "The Internet of Things in scientific literature" section contains the analysis of the literature relating to the Internet of Things. The "Results and discussion" section includes the research results, the "Conclusions" section, the final considerations, limitations and future perspectives for the study and the "Methods" section the definition of the research approach.

\section{The Internet of Things in scientific literature}

The Internet of Things (IoT) is what happens when ordinary everyday objects contain inter-connected microchips. Not only do these microchips help to keep track of other objects, but many of these devices sense their surroundings and provide information about them to other machines and to human beings. Also called M2M, standing for Machine to Machine, Machine to Man, Man to Machine or Machine to Mobile, the IoT intelligently connects humans, devices and systems.

Analysts describe two distinct modes of communication within the IoT: thing-toperson and thing-to-thing communication (Raunio 2009). Thing-to-person and personto-thing communications encompass a number of technologies and applications, wherein people interact with things and vice-versa, including remote access to objects on the part of humans, and objects that continuously report on their status, whereabouts and sensor data. Thing-to-thing communications involve technologies and applications wherein everyday objects and infrastructure interact with the human. Objects can monitor other objects, take corrective actions and notify or prompt humans as required.

Consequently, the Internet of Things (IoT) is the networked connection of people, processes, data and things that together are able to achieve more relevant and valuable connections than ever before.

This emerging global internet-based information architecture of integrated networked connections includes the existing and evolving internet and network developments.

Kevin Ashton (Johnston 2014), in 1999, was the first who, in order to describe internet-connected devices, coined the term Internet of Things. He asserted that the IoT would become able to offer advanced connectivity of devices, systems and services which, by going beyond machine-to-machine communications (M2M), cover a variety of protocols, domains and applications. This definition was followed by several other definitions provided by various authors and institutions.

The report of the International Telecommunication Union (ITU, 2005) classified the IoT as a new dimension of information and communication technology (Del Giudice and Straub, 2011).

IERC, the European Research Cluster on the Internet of Things, defines the IoT as a dynamic global network infrastructure with self-configuring capabilities based on standard 
and interoperable communication protocols where physical and virtual "things" have identities, physical attributes, and virtual personalities, use intelligent interfaces and are seamlessly integrated into the information network (Vermesan et al. 2009).

Kumar et al. (2015) assert that the evolution of the internet and related technologies has been an evolution of new paradigm, which is the Internet of Things. In this model, a large number of objects/devices are connected to one another on the internet for the purpose of sharing information, irrespective of their locations. These devices may be interconnected with one another using various network protocols and standards, in order to exchange information between each other. The underlying network used for information exchange generally has built-in intelligence, which they called ambient intelligence, so that it can make adaptive decisions concerning the exchange of information between these objects within the IoT.

Borgia (2014) has highlighted that the Internet of Things is a new model that combines aspects and technologies from different approaches: ubiquitous computing, pervasive computing, internet protocols, sensing technologies, communication technologies and embedded devices, which are merged together to form a system where the real and digital worlds meet and are continuously in symbiotic interaction. He considered smart objects as the building blocks of the IoT vision. By putting intelligence into everyday objects, they are turned into smart objects that not only can collect information from the environment and interact with/control the physical world, but also be connected to each other through the internet and exchange data and information.

Therefore, the Internet of Things is a new paradigm that can link everything with everyone within an integrated network (Rifkin 2014). Sensors and microchips can connect every aspect of economic and social life to a platform: the IoT Platform.

Every node, in the form of businesses, homes, vehicles, people, machines, natural resources, production lines, logistics networks, consumption habits, recycling flows will be linked in real-time, using sensors and software to feed into big data continuously.

Intelligent infrastructure is designed to be open, distributed and collaborative. Data collected are, therefore, processed with advanced analysis, transformed into predictive algorithms, and applied to automated systems in order to increase productivity, improve efficiency, reduce marginal costs of production and enhance quality of life.

Smart things will become active participants in business and social processes. They will interact and communicate among themselves and with people and their environment, by exchanging data and information received from the sensors. They can also react autonomously to real-world events and influence it through processes that able to trigger actions and create services with or without direct human intervention.

Intelligent infrastructure is the result of three elements that interact with each other and enable the system to operate as a whole. These elements are Energy Internet, Communications Internet and Logistics Internet.

Communications are necessary to manage economic activity, energy is needed to generate information and transport, and logistics have enabled us to move economic activity along the value chain.

The Internet of Energy ( $\mathrm{IoE}$ ), Internet of Communications ( $\mathrm{IoC}$ ) and Internet of Logistics (IoL) are part of a single operating system: the IoT platform. These three kinds of internet have created a common global IT platform of seamlessly connected networks and networked "smart objects", which are continuously finding ways to 
increase the efficiency and productivity of resources, the production and distribution of goods and services and the recycling of waste.

It suggests that there are a vast number of potential applications of the Internet of Things, in varying private and work-based domains, such as healthcare, safety, aviation, automotive, the decisional process (Lombardi et al. 2014) and the transport of people and goods and in supply chain management.

The purpose of our research is, therefore, to identify, define and highlight the main characteristics, fields of application, opportunities and issues relating to the topic of the Internet of Things.

\section{Results and discussion}

The Internet of Things (IoT) is an integrated part of the Future Internet and can be defined as a dynamic global network infrastructure with self-configuring capabilities based on standard and inter-operable communication protocols, where physical and virtual things have identities, physical attributes and virtual personalities; they use intelligent interfaces and are seamlessly integrated into the information network.

In the IoT, things are expected to become active participants in business, information and social processes, where they have the capacity to interact and communicate among themselves and with the environment, exchanging data and information that is "sensitive" to the environment, while reacting autonomously to events in the "real/physical world", which it influences by running processes that trigger actions and create services with or without direct human intervention.

Interfaces in the form of services promote interaction with these smart things over the internet, querying and changing their status and any information associated to them, all the time taking into account security and privacy issues.

Electronic sensors are now everywhere, in smartphones, cars, home electronic systems, healthcare devices, fitness monitors and in the workplace. It has been estimated that, by 2020, over 200 billion sensor devices will be inter-connected, creating a market size that, by 2025 , will be between $\$ 2.7$ trillion and $\$ 3$ trillion a year.

Smart objects can be seen as the building blocks of the IoT vision. They are not only able to collect information from the environment and interact with the physical world, but also to be interconnected, among themselves, through the internet and exchange data and information. They link people, machines, enterprises, houses, means of transport, natural resources, production lines and logistics networks, in a connected network composed of Communications Internet, Logistics Internet and Energy Internet, in a single-integrated system: the Internet of Things intelligent platform,

All of these considerations bring out the importance taken on by the IoT in the knowledge economy. There are a significant number of interconnected devices, and a remarkable amount of accessible data will be able to create new opportunities for defining services that will bring tangible benefits to society, the environment, the economy and to individual citizens.

As a consequence, the aim of this paper is to achieve an updated theory of the Internet of Things, by running through two databases (Google Scholar and EBSCO) the four key words Internet of Things, Energy Internet, Communications Internet and Logistics Internet.

The results of the research are summarised in the following Table 1. 
Table 1 Research results

\begin{tabular}{lll}
\hline Key words & Databases & \\
\cline { 2 - 3 } & EBSCO & Google scholar \\
\hline Internet of Things & 4000 & $2,310,000$ \\
Energy Internet & 386 & $3,910,000$ \\
Communications Internet & 20,165 & $3,490,000$ \\
Logistics Internet & 302 & 449,000 \\
\hline
\end{tabular}

Source: our elaboration

The table shows all the results found in the databases for each key word.

Based on the results obtained, we decided to examine the first six pages of the results for each key word, for the period of times between 1995 and 2015.

We made this choice to give more meaning to the data.

Table 2 describes the data concerning to the first database EBSCO.

We retrieved 184 scientific contributions relating to economic and corporate purposes from the EBSCO database, analysing 73 of them. Of these 73,9 were connected to the key word Internet of Things, 12 to Energy Internet, 26 to Communications Internet and 26 to Logistics Internet.

Table 3 gives the data retrieved from the second database, Google Scholar.

We found 189 scientific contributions relating to economic and business purposes on the Google Scholar database and examined 78 of them. Of these, 31 were connected to the key word Internet of Things, 36 to Energy Internet, 8 to Communications Internet and 3 to Logistics Internet. We also found three duplications.

Through the literature analysis, we were able to retrieve 480 contributions, 373 relating to economic and business purposes within the framework of the Internet of Things. The 373 scientific essays contained three duplications, meaning that had 370 scientific contributions in total, of which we read and analysed 151.

Figure 1 describes the literature production for the period analysed, according to our research.

It was possible to classify the scientific production not only on the basis of the quantitative aspect of the results, but also on the basis of the topic investigated.

Scientific production on the IoT can be applied to several domains, such as:

- Characteristics and tool

- Opportunities offered

- Regulation framework

- Issues

Table 2 EBSCO results

\begin{tabular}{lllll}
\hline Key words & Total & Duplication & Economic and corporate purposes & Available and analysed \\
\hline Internet of Things & 60 & 0 & 57 & 9 \\
Energy internet & 60 & 0 & 43 & 12 \\
Communications internet & 60 & 0 & 48 & 26 \\
Logistics internet & 60 & 0 & 36 & 26 \\
& 240 & 0 & 184 & 73 \\
\hline
\end{tabular}

Source: our elaboration 
Table 3 Google Scholar results

\begin{tabular}{lllll}
\hline Key words & Total & Duplication & Economic and corporate purposes & Available and analysed \\
\hline Internet of Things & 60 & 1 & 55 & 31 \\
Energy Internet & 60 & 1 & 48 & 36 \\
Communications Internet & 60 & 0 & 37 & 8 \\
Logistics Internet & 60 & 1 & 49 & 3 \\
& 240 & 3 & 189 & 78 \\
\hline
\end{tabular}

Source: our elaboration

- Proposal of a model

Some contributions described the IoT's characteristics and tools (Chandrakanth et al. 2014; Debasis and Jaydip 2011, Gubbi et al. 2013; Sungmin et al. 2010)

Istop Others identified the opportunities offered by the Internet of Things, such as:

- Investment opportunity. Several articles proposed a guide for investors, technology conglomerates and investment banks on how to tap into the opportunities offered by the Internet of Things. Venture capitalist, large companies and even retail investors can invest in the development of internet-connected devices. These devices are expected to penetrate in just about every category of product, from stoplights and parking spaces to jet engines and tyres (Griffith 2014);

- Expansion in the forms of communication. These can be of various types, from Human-Human to Human-Things (Capriotti and Pardo Kuklinski 2012) and Things-Things (Kumar et al. 2015; Echterhoff 2013).

- Optimisation of energy consumption. Energy-efficient devices and systems have been developed to reduce energy consumption or to optimise the network infrastructure (Aleksić 2013; Cranwell et al. 2014; Singh and Yiu 2011).

- Implications for marketing. Authors say that the IoT will be able to create product relationship marketing similar to customer relationship marketing (Benady 2014) and that it will help in analysing e-consumer behaviour (Cristóbal-Fransi et al. 2014; Malhotra 2000).

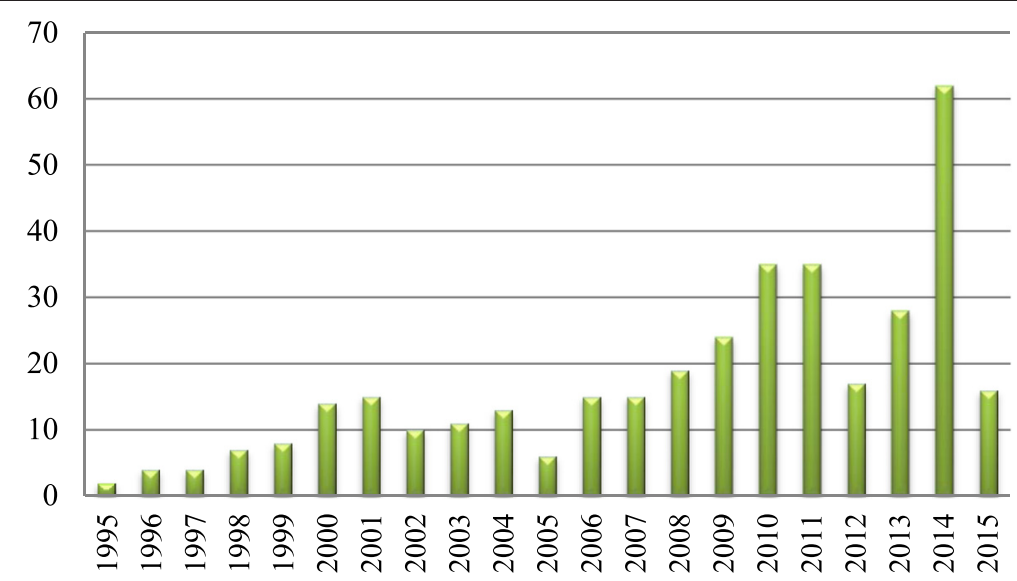

Fig. 1 The development of the Internet of Things in the analysed literature. Source: our elaboration 
- Implications for logistics (product delivery). Some authors have analysed the application of the IoT to logistics, such as product traceability (Pye 2014) or in product delivery services to ensure timely and efficient delivery to customers (Yu et al. 2015). Others discussed the characteristics of logistics in the IoT framework (Bollo and Stumm 1998) or the impact of the IoT on logistic efficiency (Shah 2008).

- Sources of innovation. The IoT could be applied in development processes, in medical technology (Wright et al. 2003), the smart city, home-control applications (Yuksekkaya et al. 2006) and strategic innovation in companies (Prince et al. 2014).

Another aspect analysed was the legal framework of the IoT. In particular, by establishing a fully operable legal framework for the Internet of Things, it should be possible to introduce the new information architecture in an effective manner. Some authors (Weber and Weber 2010) discussed the adoption of a self-regulatory approach that could provide a legal framework for the Internet of Things and, while they see this as the preferable option, others (Trequattrini et al. 2012b) prefer government regulation.

Several authors have analysed the issues relating to the IoT. These concern many features, such as privacy (Weber 2010), security (Hamad et al. 2009), ethical use of data (Roman et al. 2011), internet dependence and others psychological illnesses affecting users of this medium (Rice 2006; Ko et al. 2009).

Finally, the information from smart things has been used by authors to define and propose a model to solve the issues relating to the IoT (Atzori et al. 2011), to optimise energy management (Huang et al. 2010) or to simplify interconnection among ubiquitous things (Ning et al. 2015).

It is possible, under the previous assumption, to classify the scientific contributions we had retrieved into several domains, according to the following Table 4.

From the table, it can be seen that the main domain investigated is that of the opportunities offered by the Internet of Things, with 187 contributions; followed by characteristics and tools, with 85 contributions; issues, with 48 contributions; proposal of a model, with 40 contributions; and regulatory framework, with 10 contributions.

\section{Conclusions}

The Internet of Things will create a dynamic network of billions or trillions of wireless identifiable things communicating with one another and integrating with the developments derived from concepts like pervasive computing, ubiquitous computing and

Table 4 The contributions classified by domains

\begin{tabular}{ll}
\hline Domains & Contribution \\
\hline Characteristics and tools & 85 \\
Opportunities offered & 187 \\
Regulatory framework & 10 \\
Issues & 48 \\
Proposal of a model & 40 \\
& 370 \\
\hline
\end{tabular}

Source: our elaboration 
ambient intelligence. The Internet of Things hosts a vision of ubiquitous computing and ambient intelligence, which are enhanced by requiring, among other things, total communication and complete computing capability, and by integrating the elements of continuous communication, identification and interaction. The Internet of Things fuses the digital world and the physical world, by bringing together different concepts and technical components, so that pervasive networks, miniaturisation of devices and mobile communication all work in combination and with the new business process models (Maggioni and Del Giudice 2011).

Applications, services, middleware components, networks and endpoints will be structurally connected in entirely new ways. While we recognise that, initially, there will be commercial and physical challenges to establishing global ubiquitous network connectivity and that the many connected things and devices may have limited capacity for engaging in two-way network connectivity, it is important that the architectural design for the Internet of Things supports effective two-way caching and data synchronisation techniques. It must also support network-connected endpoints used for the virtual representations of the connected things and devices, which can be used for monitoring their location, condition and status, as well as for sending them requests and instructions.

The Internet of Things will bring tangible business benefits, such as the highresolution management of assets and products, improved life-cycle management and better collaboration between enterprises. Many of these benefits will be achieved through the unique identification of individual things, together with search and discovery services, enabling each thing to interact individually, and so build its individual life history, a record of its activities and interactions over time.

Within this framework, our aim in this the paper is to provide an updated theory on the topic of the Internet of Things, an Intelligent platform in which every node, moment to moment, in real time, is connected to the others through sensors and software.

This infrastructure is a global neutral network, designed to be open, distributed and collaborative. It can provide a broad flow of data which can be used for corporate and economic purposes.

Over the years, the importance taken on by the IoT has led many authors to investigate the topic. Therefore, in order to fully appreciate all this intelligent infrastructure's characteristics, opportunities and issues, we have carried out a qualitative and quantitative analysis of the data collected.

Table 5 describes the results of the quantitative analysis.

In reality, the study has shown that our subject is treated mainly in papers (91.3\%), then in books $(7.2 \%)$, chapters of books $(0.8 \%)$, white papers $(0.5 \%)$ and theses $(0.2 \%)$.

At the qualitative level, instead, we found that the main topic investigated was that of the opportunities offered by the Internet of Things, with 187 contributions.

Our research, therefore, highlights that the IoT is being introduced in several sectors, from the industrial to the commercial sectors (Price et al. 2013).

Table 5 The quantitative analysis results

\begin{tabular}{lllll}
\hline Papers & Books & Chapters of book & White papers & Theses \\
\hline 327 & 37 & 3 & 2 & 1 \\
\hline Source: our elaboration & & &
\end{tabular}


Companies have applied sensors to track the flow of goods and services (Bollo and Stumm 1998). Logistics companies have embedded sensors in their vehicles to monitor any potential faults, so they that can be replaced before a costly breakdown occurs (Carayannis et al. 2015).

The IoT could be used also to create smart cities. There are sensors that measure the material conditions of a city's infrastructure to assess the structural soundness of the built environment and the need for repairs; other sensors could be placed along streets to optimise driving or walking routes, monitor traffic congestion and pedestrian density, inform drivers about available parking spaces and keep drivers up-to-date on accidents and traffic delays; sensors embedded in public lighting could be used to turn lights up or down in response to the surrounding light conditions (Saint 2015).

In addition, to predict risk and determine insurance premiums and/or payouts, insurance companies are beginning to place sensors in vehicles to provide data about the time of day, week or month in which they are being used, and about the locations and distances travelled in a given period of time (Bradley et al. 2014).

The Internet of Things is being applied to the natural environment to defend the Earth's ecosystems more efficiently. Sensors are being installed across cities, suburbs and in rural communities to measure pollution levels and warn the public about toxic conditions, to minimise public exposure and protect public health; in the soil, they are used to detect subtle changes in vibrations and earth density, to inform scientists about the risk of avalanches, volcanic eruptions and earthquakes; in rivers, lakes, and oceans, they are used to report changes in the quality of water, and in wild animals and along migratory trails, they are used to evaluate environmental and behavioural changes (Del Giudice et al. 2013).

Sensors are used to monitor weather conditions, changes in moisture, the spread of pollen and other factors that affect crop yields and are transforming the way food is produced. In shipment and delivery, they are able to both track goods' whereabouts and sniff foodstuff produce to warn about product spoilage.

IoT is applied in medicine, where sensors are attached or implanted in human bodies to monitor bodily functions, including heart rate, pulse and body temperature, and can warn doctors of vital changes that could require attention.

Another application is in security and home control systems (Pang et al. 2015). Homes, offices, factories, stores and even public gathering places have been equipped with cameras and sensors to detect criminal activity and, in houses, to control home appliances.

All the same, the IoT has also raised issues concerning data security, personal privacy and health and psychological illnesses, aspects that highlight the importance of education in ethical uses of the IoT framework and the data collected.

This intelligent infrastructure, as part of the Third Industrial Revolution (Rifkin 2014), integrates the virtual environment and the natural environment within an operating network, creating synergy and encouraging interconnection, helping to avoid crises in the business world (Trequattrini et al. 2012c).

In this way, we can optimise the use, efficiency and productivity of resources, the formation and growth of new industries, the introduction of new organisational models aimed at spreading information, the production and distribution of goods and services and the recycling of waste, while ensuring the well-being of the Earth as a whole. 
The paper has some limitations, since we have only considered the literature produced between 1995 and 2015 and only the first six pages of results obtained for the two databases (Google Scholar and EBSCO).

Therefore, future analysis could be carried out to analyse a more extensive period of time and to run the key words through more databases.

\section{Methods}

The study, developed by studying national and international literature, has the aim of investigating the topic of the Internet of Things. The research approach is based on the qualitative method (Maylor and Blackmon, 2005; Myers, 2013) with the inclusion of a single method approach, and the sources of the research are of a secondary nature (documents, reports, newspapers, articles, paper and scientific books).

By analysing the existing literature over the last 20 years, the Internet of Things has been investigated by running four key words through two databases (Google Scholar and EBSCO), in order to provide a range of scientific contributions to complement our literature review. The key words applied in our research are the following:

- Internet of Things

- Energy Internet

- Communications Internet

- Logistics Internet

The data collection was carried out through a research protocol (Yin, 1994). This research protocol, required for data processing, entails carrying out several steps:

1. The elaboration of a project summary report is necessary to identify the study objective.

2. The definition of procedures linked to data acquisition and the allocation of tasks for each member of the working group. The working group consisted of five people and was involved in researching secondary data.

3. The preparation of a study report to highlight the essential points of the project.

We also defined some limits for the analysis:

- Choice of the period of time between 1995 and 2015, to restrict the research to the more recent studies.

- Analysis of the first six pages of the results for each key word, to obtain significant results.

There were 480 initial results (240 results from Google Scholar and 240 from EBSCO), and we also considered 370 scientific papers relating to the Internet of Things.

The retrieved scientific contributions are mainly articles, books or chapters of books and white papers. 
Innovation and in Public Administration Management. He had his Ph.D. in Business Administration at the University of Cassino and Southern Lazio. He is author of books and articles regarding business management, knowledge economy, intellectual capital, planning and control.

Bruno Marsigalia is a research fellow in a Business Administration in the Department of Economics and Law at the University of Cassino and Southern Lazio (Italy). His is focused on the issues of financial statements, international accounting, economic evaluations, strategic control and corporate governance. He is author of articles and books regarding extraordinary corporate transaction, corporate crisis, strategic control, evaluation of firm value. Federica Evangelista is Ph.D. student in Business Administration in the Department of Economics and Law at the University of Cassino and Southern Lazio in Italy. Her research interests cover the following topics: business administration, business management, corporate governance, corporate social responsibility, intellectual capital and intellectual property rights, intellectual asset management and knowledge management, international accounting, leadership. She's author of articles regarding corporate governance, intellectual capital and management of intangible assets.

Matteo Palmaccio was born in Formia (Italy) in 1990 where he still lives. He holds a Master Degree in Economics and Business Law (cum laude) at the University of Cassino and Southern Lazio during which he completed a period of study abroad in the LLP-Erasmus project. In the last academic year he attended a Post-Graduate Advanced Course in Corporate Insolvency Law at the University of Rome "La Sapienza". In september he has been admitted at the Ph.D program in Business Administration at the University of Cassino and Southern Lazio. His main research interests focus on corporate disclosure, intellectual capital, international accounting, corporate insolvency, business evaluation. Marina Maggioni holds a degree in Business Management at the University of Naples "Federico II" and a Phd in Entrepreneurship and Innovation received from the Second University of Naples. She is lecturer on Business Management, Marketing Management and Advanced Business Management in the University of Rome "Link Campus". Her research interests deal with business strategy studies, technology transfer, knowledge management and corporate governance.

\section{Authors' contributions}

This paper is the joint work of the five authors: the "Introduction" is by GR, the "The Internet of Things in scientific literature" paragraph is by MM, the paragraph entitled "Methods" is by BM, the "Results and discussion" paragraph is by FE and the "Conclusions" is by MP.

\section{Author details}

${ }^{1}$ Department of Business and Law, University of Cassino and Southern Lazio, Cassino, Italy. ${ }^{2}$ Link Campus University, Rome, Italy.

Received: 23 October 2015 Accepted: 7 November 2015

\section{Published online: 14 November 2015}

\section{References}

Aleksić, S. (2013). Energy-efficient communication networks for improved global energy productivity. Telecommunication Systems, 54(2), 183-199.

Atzori, L., lera, A., \& Morabito, G. (2010). The Internet of Things: a survey. Computer networks, 4(15), 2787-2805.

Atzori, L., lera, A., \& Morabito, G. (2011). SloT: giving a social structure to the Internet of Things. Communications Letters, IEEE, 15(11), 1193-1195.

Benady, D. (2014). The internet of things. Marketing, 28(3), 34-37.

Bollo, D., \& Stumm, M. (1998). Possible changes in logistic chain relationships due to Internet developments. International Transactions in Operational Research, 5(6), 427-426.

Borgia, E. (2014). The Internet of Things vision: key features, applications and open issues. Computer Communications, $54,1-31$

Bradley, T., Thibodeau, P., \& Ng, V. (2014). The Internet of Things threats and challenges. Network World Asia, 11(1), 16-18.

Capriotti, P., \& Pardo Kuklinski, H. (2012). Assessing dialogic communication through the Internet in Spanish museums. Public Relations Review, 38(4), 619-626.

Carayannis, E. G., Campbell, D. F. J., \& Rehman, S. S. (2015). "Happy accidents": innovation-driven opportunities and perspectives for development in the knowledge economy. Journal of Innovation and Entrepreneurship, 4, 7. retrieved http://link.springer.com/article/10.1186/s13731-015-0021-9/fulltext.html. Accessed 20 September 2015.

Chandrakanth, S., Venkatesh, K., Uma Mahesh, J., \& Naganjaneyulu, K. V. (2014). Internet of Things. International Journal of Innovations \& Advancement in Computer Science, 3(8), 16-20.

Cranwell, J., Benford, S., Houghton, R. J., Golembewksi, M., Fischer, J. E., \& Hagger, M. S. (2014). Increasing self-regulatory energy using an Internet-based training application delivered by smartphone technology. CyberPsychology, Behavior \& Social Networking, 17(3), 181-186.

Cristóbal-Fransi, E., Daries-Ramon, N., \& Baldomar, P. J. (2014). E-consumer segmentation: an applied study based in the internet use perspectives. Cuadernos de Gestión, 14(1), 33-55.

Debasis, B., \& Jaydip, S. (2011). Internet of Things: applications and challenges in technology and standardization. Wireless Personal Communications, 58(1), 49-69.

Del Giudice, M., Della Peruta, M. R., \& Maggioni, V. (2013). Collective knowledge and organizational routines within academic communities of practice: an empirical research on science-entrepreneurs. Journal of the Knowledge Economy, 4(3), 260-27.

Del Giudice, M., \& Straub, D. (2011). IT and entrepreneurism: an on-again, off-again love affair or a marriage? MIS Quarterly, 35(4), 3-11.

Echterhoff, G. (2013). How Communication on the Internet affects memory and shared reality: talking heads online. Psychological Inquiry, 24(4), 297-300.

Foray, D. (2004). Economics of knowledge. Boston, MA: MIT Press. 
Griffith, E. (2014). How to invest in the internet of things. Fortune, 170(9), 112-116.

Gubbi, J., Buyya, R., Marusic, S., \& Palaniswami, M. (2013). Internet of Things (loT): a vision, architectural elements, and future directions. Future Generation Computer Systems, 29(7), 1645-1660.

Hamad, F., Smalov, L., \& James, A. (2009). Energy-aware security in M-Commerce and the Internet of Things. IETE Technical Review, 26(5), 357-362.

Huang, A. Q., Crow, M. L., Heydt, G. T., Zheng, J. P., \& Dale, S. J. (2010). The future renewable electric energy delivery and management (FREEDM) system: the energy internet. Proceedings of the IEEE, 99(1), 133-148.

ITU, (2005). The Internet of Things, Internet reports 2005, available at: http://www.itu.int/wsis/tunis/newsroom/stats/ The-Internet-of-Things-2005.pdf. Accessed 1 September 2015

Johnston, W. J. (2014). The future of business and industrial marketing and needed research. Journal of Business Marketing Management, 7(1), 296-300.

Ko, C. H., Yen, J. Y., Liu, S. C., Huang, C. F., \& Yen, C. F. (2009). The associations between aggressive behaviors and Internet addiction and online activities in adolescents. Journal of Adolescent Health, 44(6), 598-605.

Kumar, N., Chilamkurti, N., \& Misra, S. (2015). Bayesian coalition game for the internet of things: an ambient intelligence-based evaluation. IEEE Communications Magazine, 53(1), 48-55.

Lombardi, R., Trequattrini, R., \& Battista, M. (2014). Systematic errors in decision making processes: the case of the Italian Serie A football championship. International Journal of Applied Decision Sciences, 7(3), 239-254.

Maggioni, V., \& Del Giudice, M. (2011). Relazioni sistemiche tra imprenditorialità interna e gemmazione d'impresa: una ricerca empirica sulla natura cognitiva delle nuove imprese. Sinergie, 71, 171-197.

Malhotra, Y. (2000). Knowledge management for e-business performance: advancing information strategy to "internet time". Information Strategy: The Executive's Journal, 16(4), 5-16.

Maylor, H., \& Blackmon, K. (2005). Researching business and management. New York: Palgrave Macmillan.

Myers, M. D. (2013). Qualitative research in business \& management. London; UK: Sage.

Ning, H., Fu, Y., Hu, S., \& Liu, H. (2015). Tree-Code modeling and addressing for non-ID physical objects in the Internet of Things. Telecommunication Systems, 58(3), 195-204.

Prince, K., Barrett, M., \& Oborn, E. (2014). Dialogical strategies for orchestrating strategic innovation networks: the case of the Internet of Things. Information \& Organization, 24(2), 106-127.

Pye, A. (2014). The internet of things connecting the unconnected. Engineering \& Technology, 9(11), 64-70.

Raunio, B, (2009). The Internet of Things, A report from the November 5, 2009 seminar, .SE:s Internet guide, No. 16, - English edition, Version 1.0, Sweden, https://www.iis.se/docs/The-Internet-of-things.pdf. Accessed on 12 January 2015

Rifkin, J. (2014). The zero marginal cost society: the Internet of Things, the collaborative commons, and the eclipse of capitalism. New York: Palgrave MacMillan.

Roman, R., Najera, P., \& Lopez, J. (2011). Securing the Internet of Things. Computer, 44(9), 51-58,

Rice, R. E. (2006). Influences, usage, and outcomes of Internet health information searching: multivariate results from the Pew surveys. International Journal of Medical Informatics, 75(1), 8-28.

Pang, Z., Zheng, L., Tian, J., Kao-Walter, S., Dubrova, E., \& Chen, Q. (2015). Design of a terminal solution for integration of in-home health care devices and services towards the Internet-of-Things. Enterprise Information Systems, 9(1), 86-116.

Price, D. P., Stoica, M., \& Boncella, R. J. (2013). The relationship between innovation, knowledge, and performance in family and non-family firms: an analysis of SMEs. Journal of Innovation and Entrepreneurship, 2, 14. retrieved http://www.innovation-entrepreneurship.com/content/pdf/2192-5372-2-14.pdf. Accessed 3 September 2015

Shah, P. (2008). Logistics websploitation. Supply Chain Europe, 17(6), 46-47.

Singh, S., \& Yiu, C. (2011). Putting the cart before the horse: merging traffic for energy conservation. IEEE Communications Magazine, 49(6), 78-82.

Saint, A. (2015). Where next for the Internet of Things? Engineering \& Technology, 10(1), 72-75.

Sungmin, H., Daeyoung, K., Minkeun, H., \& Sungho, B. (2010). SNAIL: an IP-based wireless sensor network approach to the internet of things. Wireless Communications, IEEE, 17(6), 34-42.

Trequattrini, R., Russo, G., \& Lombardi, R. (2012a). Defining business network. International Journal of Business Research and Management, 3(1), 29-34.

Trequattrini, R., Russo, G., \& Lombardi, R. (2012b). Network governance: organisational and legal profiles. Corporate Ownership \& Control, 9(4), 346-350.

Trequattrini, R., Russo, G., \& Lombardi, R. (2012c). The crisis of company networks and tools for its prediction. International Business Research, 5(10), 46-55.

Yin, R. (1994). Case study Research. Thousand Oaks, CA: Sage Publication.

Yuksekkaya, B., Kayalar, A. A., Tosun, M. B., Ozcan, M. K., \& Alkar, A. Z. (2006). A GSM, internet and speech controlled wireless interactive home automation system consumer electronics. IEEE Transaction, 52(3), 837-843.

Yu, J., Subramanian, N., Ning, K., \& Edwards, D. (2015). Product delivery service provider selection and customer satisfaction in the era of internet of things: a Chinese e-retailers' perspective. International Journal of Production Economics, 159, 104-116.

Vermesan, O., Friess, P., Guillemin, P., Gusmeroli, S., Sundmaeker, H., Bassi, A., et al. (2009). Internet of Things strategic research roadmap. Brussels: European Commission-Information Society and Media DG.

Weber, R. H. (2010). Internet of Things—new security and privacy challenges. Computer Law \& Security Review, 26(1), $23-30$.

Weber, R. H., \& Weber, R. (2010). Internet of Things. Legal Perspectives, New York, NY: Springer

Wright, K. B., Bell, S. B., Wright, K. B., \& Bell, S. B. (2003). Health-related support groups on the Internet: linking empirical findings to social support and computer-mediated communication theory. Journal Health Psychology, 8(1), 39-54. 